\title{
Effect of Working Memory on Students' Attitude towards Mathematics at Secondary Level
}

\author{
Asim Nazir* \\ Rizwan Akram Rana ** \\ Khateeb Ahmed Khan ${ }^{* * * *}$
}

\begin{abstract}
The aim of this study was to find out the influence of working memory on secondary school students' attitude towards mathematics. The sample of the study was comprised of 1303 students of age 14-15. Digit backward test was used to measure the working memory capacity of the students while questionnaire was used for the measurement of their attitude towards mathematics. Data were analyzed using the chi-square test to find the effect of working memory capacity on students' attitude towards mathematics at secondary level. Independent sample t-test was used to find the difference between students' working memory capacity based on gender, grades, school type, residential area, and elective subjects. Results suggest that the working memory of the students is consistent with their age. Female students have higher working memory capacity than male students. Urban students have higher memory than rural students. Overall results revealed that students with higher working memory have better attitude towards mathematics than the students with low working memory capacity at secondary level. It was recommended that there is a need of giving attention towards the working memory capacity of students for developing positive attitude towards mathematics.
\end{abstract}

Keywords: working memory capacity, attitude, digit backward test

\footnotetext{
${ }^{*}$ Lecturer and Ph.D Scholar, Institute of Education and Research, University of the Punjab, Lahore, Pakistan, Email: asimierpu@gmail.com

${ }^{* *}$ Professor, Institute of Education and Research, University of the Punjab, Lahore, Pakistan, Email: drrarpu@ gmail.com

${ }^{* * *}$ Ph.D Scholar, Institute of Education and Research, University of the Punjab, Lahore, Pakistan, Email: khateeb.pu@gmail.com
} 


\section{Introduction}

Atkinson and Shiffrin's (1968) proposed a model which is also termed as Atkinson and Shiffrin Model on working memory. This model got fame also with the names of multi-store model and modal model. Atkinson and Shiffrin's model led to the dissatisfaction with regular short-term memory proposed by James (1890, 1970). Baddeley and Hitch (1974) suggested their own multi-component model termed as working memory. Working memory is a structured model of memory which provides temporary storage of the information and its manipulation which is vital for creating essential relationship between controlled action and perception (Alenezi, 2008). Johnstone (1984) defined working memory by referring the brain as that part of body where information can be held, processed, organized, and shaped prior to its storage in long-term memory for future use. Today this model of memory is most widely used and accepted one (Sternberg \& Sternberg, 2012).

Baddeley and Hitch (1974) conceived working memory as a multicomponent system which consisted of three parts: (i) the central executive which is supported by two other self-regulating systems (ii) the phonological loop and (iii) the visuo-spatial sketchpad which are used to temporarily store information in verbal and visuo-spatial form. Central executive system is considered as the most important component of the model of working memory as it is used to divide the resources between the two components: phonological loop and visuo-spatial sketchpad. The central executive is multi-functional and complex (Bull \& Espy, 2006). The language-based information is processed in phonological loop which is a slave system of working memory. The other slave system of working memory is the visuo-spatial sketchpad which is used to process visual and spatial information.

Baddeley (1990a, 1990b, 2007, and 2009) was proposed an integrative model of memory. This integrative model of memory is an extension of working memory rather than a replacement of it. He introduced the episodic buffer in working memory. The episodic buffer is used to bind the information collected from the visuospatial sketchpad, the phonological loop, and long-term memory. This bound information is then represented as a unitary episode. The episodic buffer integrates information collected from visual-spatial sketchpad and phonological loop and convert this information into a sensible and understandable one. This 
integration of information is then used to solve the problems by evaluating the previous experiences and fresh data (Sternberg \& Sternberg, 2012).

The role of working memory in learning processes was identified by many cognitive psychologists. These psychologists supported their claims by conducting the studies which demonstrated close links among working memory capacity and measures of learning and academic achievement. Adams and Hitch (1997) found relationship between mental arithmetic and phonological loop. Mclean and Hitch (1999) also found a relationship between working memory capacity and learning in mathematics. Bull and Scerif (2001) observed positive relationship between functioning of central executive and mathematical skills. Christou (2001) observed a good relationship between working memory capacity and mathematics achievement. Towse and Houston (2001) also studied on the phonological loop and mathematics achievement. Jarvis and Gathercole (2003) pointed out the significance of visuo-spatial ability in children's mathematics performance. Alenezi (2004) worked on Kuwaiti students' working memory capacity. Holmes \& Adams (2006) proposed that the central executive plays a crucial part while calculating. In a randomized control study, Swanson (2016) found that children having high working memory capacity outperformed the children having low WMC on all measures of cognitive strategies. Simms, Frausel, and Richland (2018) showed that children's working memory capacity predicts analogical performance of students.

Attitude towards mathematics is considered as a feeling towards mathematics which can be positive or negative (McLeod, 1994) and this feeling changes with time (Rubinstein, 1986). Studies show that the attitude toward mathematics is positive when a child starts school. This attitude deteriorates as the school years pass on (Ma \& Kishor 1997). Attitudes which are developed in one's early life are not modified easily unless one has some significant experiences. The change in attitude is observed when we learn the things we understand (Deringol-Karatas, Yavuz, \& Arslan, 2014). Since education is considered as the agent of changing attitudes so it is very important for teachers to know their students' attitude not only towards the courses they are learning but also towards the daily social phenomenon. Teachers should also have the art of measuring their students' attitude so that the quality of education might be increased. In the recent years, studies measuring students' attitudes towards certain subjects of a course have gained significant importance. 
Measuring the attitude has become very vital in the field of education as relationship is observed between students' performance and their attitude towards mathematics (Marchis, 2013; Mohd, Mahmood \& Ismail, 2011). Students with a positive attitude towards Mathematics have better problem solving skills and like more to solve non-routine problems (Marchis, 2013).

Jung and Reid (2009) investigated relationship between working memory capacity and attitude towards science. They found a positive relationship between the two variables. Their study also revealed that students with higher working memory relied on understanding the concepts while students with low working memory tend rely on memorizing the concepts. But the worth mentioning thing here is that both of them wanted to understand the concepts. We conclude from the study that negative attitudes toward learning is related to failure to understand the concept.

Teachers make a huge impact on the attitude of their students (Johnstone \& Reid, 1981; Ponte et al., 1991; Reid \& Skrybina, 2002). Brown et al. (2008) conducted a study on students' choice of subject. The sample consisted of 1500 students from 17 schools of England. The students were very close to the time of their curriculum choice. At that time their attitude towards mathematics was investigated. The results revealed that the perceived difficulty was the main reason for opting out their subjects. This perception of difficulty might be coming from outside which suggests them that their future in mathematics might be a struggle. The feeling of not coping with mathematics might then be generated due to this perception (Kyriacou \& Goulding, 2006; Matrhews \& Pepper, 2005).

The choice of subject depends also upon the region also. For example, in Scotland, the reason for adopting mathematics for higher education is that mathematics is perceived as an essential part of overall education. While in England, a major motive for adopting mathematics is to become a mathematician or to select a career deeply reliant on mathematics (Ali \& Reid, 2012). People do not take interest in mathematics as they consider it a boring subject (Matthews \& Pepper, 2005). This is similar to the situation here in Pakistan where mathematics is considered as the most difficult subject, but students have to learn it compulsory. The results from Punjab Examination Commission (PEC) report show that performance of 
students in mathematics is the lowest i.e. $45 \%$ as compared to other subjects (Pakistan Alliance for Maths and Science, 2017).

As working memory totally deals with the retention of chunks of information in the short-term memory for the sake of processing so does the mathematics. This refers that working memory may contribute in developing attitude towards mathematics among students. Working memory capacity has been revealed as an imperative aspect while controlling the understanding of science subjects. As far as the success in learning of sciences is concerned, the attitudes related studies are also known to be very important. Working memory capacity can be linked with positive attitude if we consider it as a rate controlling feature of learning and success in understanding which leads to more positive attitudes (Jung \& Reid, 2009).

Alenezi (2008) found a positive relationship between the working memory capacity and attitude towards mathematics. They compared the attitude of students with their age level (11-12). Ali and Reid (2012) compared the working memory of students with their grade level (grade 5, $6,7)$. They also compared the working memory of Urdu medium students and English medium students. Their study did not compare students' attitude towards mathematics on the basis of their categories of working memory capacity. This study compares the attitude of students towards mathematics with respect to working memory on the basis of its categories (low, average, high). The study also focuses on the students of higher age level (15-16) as compared to the previous researches which were mainly focused on lower age levels. This study will also be helpful in perceiving that the working memory can also effect the attitude of students towards mathematics. So the objective of this study was to find out the effect of working memory capacity on secondary school students' attitude towards mathematics. The study also aims to investigate secondary school students' working memory capacity and attitude towards mathematics, and compare the secondary school students' working memory capacity on the basis of their gender, grades, school type, residential area, and subject choice.

\section{Research Questions}

Following were the research questions of the study.

i. How much is the working memory capacity of secondary school science students?

ii. What is the level of secondary school science students' attitude towards mathematics? 
iii. Is there any significant mean difference between secondary school science students' working memory capacity on the basis of their gender, grades, school type, residential area, and subject choice?

iv. Is there any difference between secondary school science students' attitude towards mathematics on the basis of their working memory capacity?

\section{Methodology}

This study was quantitative in nature and casual comparative method was used to conduct this study. Cross-sectional survey design was used to collect the data and explore the impact of secondary school science students' working memory capacity on their attitude towards mathematics. Population of the study was comprised of public and private secondary schools of district Lahore. Stratified cluster sampling technique was used to draw a sample from the population. The Lahore district was divided into 5 tehsils for this purpose. Within each tehsil, 4 public and 2 private schools were randomly selected. Male and female science students of grades 9 and 10 in these schools were the sample of the study. So, the sample was comprised of 1303 secondary school science students from 30 schools. Out of 1303 students, 419 were male while 884 were female students. 719 students were studying Biology as elective science subject while 584 were studying computer as elective subject in science group.

A survey questionnaire developed by Alenezi (2008) was used to assess the attitude of students towards mathematics. The attitude scale was translated into Urdu. The translated version of the questionnaire was validated from the experts of science and mathematics education. The questionnaire was improved in the light of recommendations of the experts. The questionnaire was then piloted on 100 secondary school science students of district Lahore. After validation and pilot testing the attitude measurement questionnaire was finally comprised of seven parts measuring different aspects of attitude: i) attitude and feeling in general about mathematics, ii) attitude towards learning mathematics, iii) mathematics preference among other subjects, iv) attitude towards different topic, v) opinion about mathematics as a subject, vi) opinion about mathematics lessons, and vii) opinion about mathematics test and examination. These aspects were measured on different scales and hence treated separately. There were two types of scales. One was the Likert type scale (ranging from 1 to 5) and the other was semantic differential scale (ranging from 1 to 6). Parts i, vi, and vii were measured on Likert type 
scales and their Cronbach alpha $(\alpha)$ reliability coefficient was 0.82 . Parts ii, iii, iv, and $v$ were measured on semantic differential scale and their Cronbach alpha $(\alpha)$ reliability coefficient was 0.73 .

To measure the working memory capacity of students, Digit Backward Test (DBT) was used. DBT was comprised of 14 series of digits starting from a series of 2 digits and ending on a series of 8 digits. Each series was repeated twice e.g. there were two series of 4 digits (2547 and 5019). The researcher had to speak aloud the digits in the series with the break of 1 second between each digit. As the researcher finished speaking a complete series then the students started writing the spoken words. Students' working memory was measured by counting the series which were written correctly with no mistake. As soon as there occurred two consecutive mistakes, while the test progressed, the counting of series was stopped. For example, if a student wrote 4-digit series correctly and the next two series of 5 digits were incorrect then the working memory will be 4 despite any correct upcoming series as shown in figure 1 . The test was time bound and the students had to complete it within a specified time.

\begin{tabular}{|c|c|c|c|c|c|c|}
\hline $\begin{array}{l}\text { Working } \\
\text { Memory }\end{array}$ & \multicolumn{6}{|c|}{ Series } \\
\hline \multirow{2}{*}{2} & 4 & 2 & & & & $\checkmark$ \\
\hline & 8 & 5 & & & & $\checkmark$ \\
\hline \multirow{2}{*}{3} & 9 & 2 & 6 & & & $\checkmark$ \\
\hline & 5 & 1 & 4 & & & $\checkmark$ \\
\hline \multirow{2}{*}{4} & 2 & 5 & 7 & 4 & & $x$ \\
\hline & 5 & 0 & 1 & 9 & & $\checkmark$ \\
\hline \multirow{2}{*}{5} & 8 & 1 & 4 & 3 & 1 & $x$ \\
\hline & 6 & 5 & 8 & 4 & 2 & $x$ \\
\hline
\end{tabular}

Figure 1: A Sample of the Digit Backward Test

\section{Results of the Study}

After collecting the data, it was organized and summarized. Both descriptive and inferential techniques were applied to analyze the data. To summarize the data, mean, standard deviation, percentage, and frequencies 
were measured as descriptive statistics. Chi square and t-Test were used as inferential statistics to draw inferences.

The results of the students working memory capacity are shown in table 1 to answer the $1^{\text {st }}$ research question. The results about working memory capacity showed that the mean of the scores of secondary school students' (average age 14-15 years) working memory capacity is 6.68 which is consistent with the established rules of this age (Alenezi, 2008).

Table 1

Classification of Students' Working Memory Capacity

\begin{tabular}{llcccc}
\hline $\begin{array}{l}\text { Working } \\
\text { Memory } \\
\text { Classes }\end{array}$ & \multicolumn{1}{c}{ Groups } & Frequencies & Percentages & Mean & SD \\
\hline Up to 4 & $\begin{array}{l}\text { Low Working } \\
\text { Memory }\end{array}$ & 205 & $15.7 \%$ & 2.92 & 0.85 \\
5 & $\begin{array}{l}\text { Average Working } \\
\text { Memory }\end{array}$ & 118 & $9.1 \%$ & 5.00 & 0.00 \\
& High Working & 980 & $75.2 \%$ & 7.67 & 0.65 \\
$\begin{array}{l}\text { and } \\
\text { above }\end{array}$ & Memory & 1303 & $100 \%$ & 6.68 & 0.75 \\
Total & & & & & \\
\hline
\end{tabular}

The students were divided into three groups on the basis of their working memory capacity. The students with working memory up to 4 were classified as low working memory students (Mean $=2.92$ ). The students with working memory capacity 5 were classified as average working memory students (Mean $=5$ ) and the students with working memory capacity 6 and above were classified as high working memory students (Mean $=7.67$ ). Results showed that $75.2 \%$ students had high working memory capacity at secondary level.

Independent sample t-test was conducted to answer the $3^{\text {rd }}$ research question. The results of the independent sample t-test revealed that t-value is significant for gender $(\mathrm{t}=3.41, \mathrm{p}=0.001)$, residential area $(\mathrm{t}=3.54$, $\mathrm{p}=0.000)$ and for subject choice $(\mathrm{t}=2.25, \mathrm{p}=0.025)$. While the $\mathrm{t}$-value is not significant for grades $(\mathrm{t}=1.62, \mathrm{p}=0.106)$ and school type $(\mathrm{t}=0.65$, $\mathrm{p}=0.517$ ). So we can conclude that working memory capacity of male and female students, urban and rural students, and Biology and computer 
science students differ significantly at secondary level. The results are shown in table 2.

Further mean values from table 2 show that the working memory capacity of female students (Mean=6.81, $\mathrm{SD}=1.84$ ) is higher than the male secondary school students. Also the working memory capacity of urban (Mean $=6.80$, $\mathrm{SD}=1.84$ ) secondary school students is better than the rural (Mean=6.39, $\mathrm{SD}=2.03$ ) secondary school students. While the working memory capacity of the biology students (Mean=6.78, $\mathrm{SD}=1.85$ ) is greater than the computer science students (Mean=6.55, $\mathrm{SD}=1.97$ ) at secondary level.

Table 2

Independent sample t-test for mean difference among students' working memory capacity on the basis of gender, grades, school type, residential area, and elective subject

\begin{tabular}{lcccccc}
\hline \multicolumn{2}{c}{ Variables } & $\mathrm{N}$ & Mean & $\begin{array}{c}\text { Std. } \\
\text { Deviation }\end{array}$ & $\mathrm{t}$ & $\begin{array}{c}\mathrm{p}- \\
\text { value }\end{array}$ \\
\hline Gender & Male & 419 & 6.42 & 2.04 & 3.41 & 0.001 \\
Grades & Female & 884 & 6.81 & 1.84 & & \\
& $9^{\text {th }}$ & 610 & 6.59 & 1.98 & 1.62 & 0.106 \\
Schools & $10^{\text {th }}$ & 693 & 6.76 & 1.84 & & \\
Residence & Public & 930 & 6.70 & 1.92 & 0.65 & 0.517 \\
& Private & 373 & 6.63 & 1.89 & & \\
Elective & Rural & 922 & 6.80 & 1.84 & 3.54 & 0.000 \\
Subject & Biology & 719 & 6.39 & 2.03 & & \\
& Computer & 584 & 6.78 & 1.85 & 2.25 & 0.025 \\
& Science & 58 & 1.97 & &
\end{tabular}

Students' attitude towards mathematics was investigated and compared on the basis of their working memory capacity by using frequencies, percentages and chi square in order to answer the research questions $2^{\text {nd }}$ and $4^{\text {th }}$ as shown in tables $3,4,5,6,7,8$, and 9 .

In table 3, students' attitude and feelings in general about mathematics are compared. Results revealed that there was a significant difference between students' opinion about enjoying mathematics lessons $\left(\chi^{2}=17.71\right.$, $\mathrm{p}=0.024)$, opinion that someone must have born with the right kind of brain, to be good in mathematic $\left(\chi^{2}=18.07, \mathrm{p}=0.021\right)$, thinking that mathematics is a useful subject $\left(\chi^{2}=27.41, \mathrm{p}=0.001\right)$ and perception that mathematics knowledge is useful in daily life $\left(\chi^{2}=20.31, \mathrm{p}=0.009\right)$. Further the comparison of frequencies and percentages of high, average and low working memory capacity of the students revealed that the students with high working memory capacity $(35.53 \%)$ enjoy mathematics more than the students with low $(6.75 \%)$ working memory capacity. Also $62.7 \%$ high working memory capacity students think that if someone 
wants to be good in mathematics then he/ she must have a right kind of brain. Similarly, $60.32 \%$ students with high working memory capacity think that mathematics is a useful subject which is greater than the average $(6.53 \%)$ and low (11.71\%) working memory capacity students. Also $58.17 \%$ high working memory capacity students feel that mathematics knowledge is useful in daily life which is greater than average working memory capacity $(6.53 \%)$ and low working memory capacity students $(10.74 \%)$. Table 3 also shows that there is no significant difference between science students opinion about understanding mathematics ideas easily $\left(\chi^{2}=13.28, p=0.102\right)$, everyone should learn mathematics at secondary school $\left(\chi^{2}=14.36, \mathrm{p}=0.073\right)$, think that they are good in mathematics $\left(\chi^{2}=7.59, \mathrm{p}=0.474\right)$ and the opinion to spend more time for studying mathematics $\left(\chi^{2}=14.83, \mathrm{p}=0.063\right)$.

Table 3

Chi-square for the comparison of students' attitude and feeling in general about mathematics on the basis of their working memory capacity

\begin{tabular}{|c|c|c|c|c|c|c|c|c|c|}
\hline Statement & $\begin{array}{l}\text { Working } \\
\text { Memory }\end{array}$ & SA & $A$ & $\mathrm{~N}$ & $D$ & SD & $\chi^{2}$ & $\mathrm{df}$ & $\begin{array}{c}\mathrm{p}- \\
\text { value }\end{array}$ \\
\hline $\begin{array}{l}\text { I usually } \\
\text { understand } \\
\text { mathematics } \\
\text { idea easily }\end{array}$ & $\begin{array}{c}\text { High } \\
\text { Average } \\
\text { Low }\end{array}$ & $\begin{array}{c}264 \\
20.26 \% \\
27 \\
2.07 \% \\
45 \\
3.45 \%\end{array}$ & $\begin{array}{c}471 \\
36.15 \% \\
52 \\
3.99 \% \\
100 \\
7.67 \%\end{array}$ & $\begin{array}{c}96 \\
7.37 \% \\
15 \\
1.15 \% \\
24 \\
1.84 \%\end{array}$ & $\begin{array}{c}103 \\
7.90 \% \\
12 \\
0.92 \% \\
18 \\
1.38 \%\end{array}$ & $\begin{array}{c}46 \\
3.53 \% \\
12 \\
0.92 \% \\
18 \\
1.38 \%\end{array}$ & 13.28 & 8 & 0.102 \\
\hline $\begin{array}{l}\text { I do not enjoy } \\
\text { mathematics } \\
\text { lessons }\end{array}$ & $\begin{array}{c}\text { High } \\
\text { Average } \\
\text { Low }\end{array}$ & $\begin{array}{c}114 \\
8.75 \% \\
15 \\
1.15 \% \\
34 \\
2.61 \%\end{array}$ & $\begin{array}{c}231 \\
17.73 \% \\
15 \\
1.15 \% \\
46 \\
3.53 \%\end{array}$ & $\begin{array}{c}172 \\
13.20 \% \\
20 \\
1.53 \% \\
37 \\
2.84 \%\end{array}$ & $\begin{array}{c}240 \\
18.42 \% \\
40 \\
3.07 \% \\
57 \\
4.37 \%\end{array}$ & $\begin{array}{c}223 \\
17.11 \% \\
28 \\
2.15 \% \\
31 \\
2.38 \%\end{array}$ & 17.71 & 8 & 0.024 \\
\hline $\begin{array}{l}\text { I think everyone } \\
\text { should learn } \\
\text { mathematics at } \\
\text { secondary } \\
\text { school }\end{array}$ & $\begin{array}{c}\text { High } \\
\text { Average } \\
\text { Low }\end{array}$ & $\begin{array}{c}326 \\
25.02 \% \\
36 \\
2.76 \% \\
64 \\
4.91 \%\end{array}$ & $\begin{array}{c}366 \\
28.09 \% \\
42 \\
3.22 \% \\
69 \\
5.30 \%\end{array}$ & $\begin{array}{c}138 \\
10.59 \% \\
20 \\
1.53 \% \\
45 \\
3.45 \%\end{array}$ & $\begin{array}{c}98 \\
7.52 \% \\
11 \\
0.84 \% \\
11 \\
0.84 \%\end{array}$ & $\begin{array}{c}52 \\
3.99 \% \\
9 \\
0.69 \% \\
16 \\
1.23 \%\end{array}$ & 14.36 & 8 & 0.073 \\
\hline $\begin{array}{l}\text { I think I am } \\
\text { good in } \\
\text { mathematics }\end{array}$ & $\begin{array}{c}\text { High } \\
\text { Average } \\
\text { Low }\end{array}$ & $\begin{array}{c}239 \\
18.34 \% \\
20 \\
1.53 \% \\
36 \\
2.76 \%\end{array}$ & $\begin{array}{c}397 \\
30.47 \% \\
53 \\
4.07 \% \\
91 \\
6.98 \%\end{array}$ & $\begin{array}{c}170 \\
13.05 \% \\
22 \\
1.69 \% \\
41 \\
3.15 \%\end{array}$ & $\begin{array}{c}124 \\
9.52 \% \\
15 \\
1.15 \% \\
26 \\
2.00 \%\end{array}$ & $\begin{array}{c}50 \\
3.84 \% \\
8 \\
0.61 \% \\
11 \\
0.84 \%\end{array}$ & 7.59 & 8 & 0.474 \\
\hline
\end{tabular}




\begin{tabular}{|c|c|c|c|c|c|c|c|c|c|}
\hline $\begin{array}{l}\text { You must have } \\
\text { born with the } \\
\text { right kind of } \\
\text { brain, to be } \\
\text { good in } \\
\text { mathematic }\end{array}$ & $\begin{array}{c}\text { High } \\
\text { Average } \\
\text { Low }\end{array}$ & $\begin{array}{c}489 \\
37.53 \% \\
53 \\
4.07 \% \\
100 \\
7.67 \%\end{array}$ & $\begin{array}{c}328 \\
25.17 \% \\
41 \\
3.15 \% \\
58 \\
4.45 \%\end{array}$ & $\begin{array}{c}61 \\
4.68 \% \\
7 \\
0.54 \% \\
15 \\
1.15 \%\end{array}$ & $\begin{array}{c}59 \\
4.53 \% \\
4 \\
0.31 \% \\
12 \\
0.92 \%\end{array}$ & $\begin{array}{c}43 \\
3.30 \% \\
13 \\
1.00 \% \\
20 \\
1.53 \%\end{array}$ & 18.07 & 8 & 0.021 \\
\hline $\begin{array}{l}\text { To be good in } \\
\text { mathematics, } \\
\text { you should } \\
\text { spend more } \\
\text { time studying it }\end{array}$ & $\begin{array}{c}\text { High } \\
\text { Average } \\
\text { Low }\end{array}$ & $\begin{array}{c}541 \\
41.52 \% \\
64 \\
4.91 \% \\
107 \\
8.21 \%\end{array}$ & $\begin{array}{c}328 \\
25.17 \% \\
38 \\
2.92 \% \\
59 \\
4.53 \%\end{array}$ & $\begin{array}{c}40 \\
3.07 \% \\
2 \\
0.15 \% \\
15 \\
1.15 \%\end{array}$ & $\begin{array}{c}33 \\
2.53 \% \\
7 \\
0.54 \% \\
14 \\
1.07 \%\end{array}$ & $\begin{array}{c}38 \\
2.92 \% \\
7 \\
0.54 \% \\
10 \\
0.77 \%\end{array}$ & 14.83 & 8 & 0.063 \\
\hline $\begin{array}{l}\text { I think } \\
\text { mathematics is } \\
\text { useful subject }\end{array}$ & $\begin{array}{c}\text { High } \\
\text { Average } \\
\text { Low }\end{array}$ & $\begin{array}{c}397 \\
30.47 \% \\
35 \\
2.69 \% \\
71 \\
5.45 \%\end{array}$ & $\begin{array}{c}389 \\
29.85 \% \\
50 \\
3.84 \% \\
82 \\
6.29 \%\end{array}$ & $\begin{array}{c}88 \\
6.75 \% \\
16 \\
1.23 \% \\
32 \\
2.46 \%\end{array}$ & $\begin{array}{c}70 \\
5.37 \% \\
6 \\
0.46 \% \\
6 \\
0.46 \%\end{array}$ & $\begin{array}{c}36 \\
2.76 \% \\
11 \\
0.84 \% \\
14 \\
1.07 \%\end{array}$ & 27.41 & 8 & 0.001 \\
\hline $\begin{array}{l}\text { I find my } \\
\text { mathematics } \\
\text { knowledge } \\
\text { useful in daily } \\
\text { life }\end{array}$ & $\begin{array}{c}\text { Average } \\
\text { Low }\end{array}$ & $\begin{array}{c}389 \\
29.85 \% \\
38 \\
2.92 \% \\
77 \\
5.91 \%\end{array}$ & $\begin{array}{c}369 \\
28.32 \% \\
47 \\
3.61 \% \\
63 \\
4.83 \%\end{array}$ & $\begin{array}{c}107 \\
8.21 \% \\
9 \\
0.69 \% \\
37 \\
2.84 \%\end{array}$ & $\begin{array}{c}56 \\
4.30 \% \\
10 \\
0.77 \% \\
12 \\
0.92 \%\end{array}$ & $\begin{array}{c}59 \\
4.53 \% \\
14 \\
1.07 \% \\
16 \\
1.23 \%\end{array}$ & 20.31 & 8 & 0.009 \\
\hline
\end{tabular}

Table 4

Chi-square for the comparison of students' attitude towards learning mathematics on the basis of their working memory capacity

\begin{tabular}{|c|c|c|c|c|c|c|c|c|c|c|}
\hline Statement & $\begin{array}{l}\text { Working } \\
\text { Memory }\end{array}$ & & & $\begin{array}{r}\text { Frequer } \\
\%\end{array}$ & cies & & & $\chi^{2}$ & $d f$ & $\begin{array}{c}\text { p- } \\
\text { value }\end{array}$ \\
\hline $\begin{array}{l}\text { I am } \\
\text { confident in } \\
\text { mathematics } \\
\text { classes }\end{array}$ & $\begin{array}{c}\text { High } \\
\text { Average } \\
\text { Low }\end{array}$ & $\begin{array}{c}551 \\
42.29 \% \\
58 \\
4.45 \% \\
114 \\
8.75 \%\end{array}$ & $\begin{array}{c}210 \\
16.12 \% \\
28 \\
2.15 \% \\
43 \\
3.30 \%\end{array}$ & $\begin{array}{c}69 \\
5.30 \% \\
6 \\
0.46 \% \\
22 \\
1.69 \%\end{array}$ & $\begin{array}{c}35 \\
2.69 \% \\
4 \\
0.31 \% \\
10 \\
0.77 \%\end{array}$ & $\begin{array}{c}22 \\
1.69 \% \\
7 \\
0.54 \% \\
9 \\
0.69 \%\end{array}$ & $\begin{array}{c}93 \\
7.14 \% \\
15 \\
1.15 \% \\
7 \\
0.54 \%\end{array}$ & 22.17 & 10 & 0.014 \\
\hline $\begin{array}{l}\text { Mathematics } \\
\text { is too } \\
\text { abstract for } \\
\text { me }\end{array}$ & $\begin{array}{c}\text { High } \\
\text { Average } \\
\text { Low }\end{array}$ & $\begin{array}{c}276 \\
21.18 \% \\
23 \\
1.77 \% \\
56 \\
4.30 \%\end{array}$ & $\begin{array}{c}131 \\
10.05 \% \\
16 \\
1.23 \% \\
23 \\
1.77 \%\end{array}$ & $\begin{array}{c}69 \\
5.30 \% \\
4 \\
0.31 \% \\
19 \\
1.46 \%\end{array}$ & $\begin{array}{c}75 \\
5.76 \% \\
18 \\
1.38 \% \\
24 \\
1.84 \%\end{array}$ & $\begin{array}{c}133 \\
10.21 \% \\
21 \\
1.61 \% \\
38 \\
2.92 \%\end{array}$ & $\begin{array}{c}296 \\
22.72 \% \\
36 \\
2.76 \% \\
45 \\
3.45 \%\end{array}$ & 23.76 & 10 & 0.008 \\
\hline
\end{tabular}




\begin{tabular}{|c|c|c|c|c|c|c|c|c|c|c|}
\hline $\begin{array}{l}\text { I am getting } \\
\text { worse at }\end{array}$ & High & $\begin{array}{c}139 \\
10.67 \%\end{array}$ & $\begin{array}{c}55 \\
4.22 \%\end{array}$ & $\begin{array}{c}68 \\
5.22 \%\end{array}$ & $\begin{array}{c}73 \\
5.60 \%\end{array}$ & $\begin{array}{c}129 \\
9.90 \%\end{array}$ & $\begin{array}{c}516 \\
39.60 \%\end{array}$ & 30.68 & 10 & 0.001 \\
\hline mathematics & $\begin{array}{c}\text { Average } \\
\text { Low }\end{array}$ & $\begin{array}{c}16 \\
1.23 \% \\
29 \\
2.23 \%\end{array}$ & $\begin{array}{c}9 \\
0.69 \% \\
18 \\
1.38 \%\end{array}$ & $\begin{array}{c}22 \\
1.69 \% \\
28 \\
2.15 \%\end{array}$ & $\begin{array}{c}6 \\
0.46 \% \\
14 \\
1.07 \%\end{array}$ & $\begin{array}{c}17 \\
1.30 \% \\
26 \\
2.00 \%\end{array}$ & $\begin{array}{c}48 \\
3.68 \% \\
90 \\
6.91 \%\end{array}$ & & & \\
\hline $\begin{array}{l}\text { I feel I am } \\
\text { coping well }\end{array}$ & $\begin{array}{c}\text { High } \\
\text { Average } \\
\text { Low }\end{array}$ & $\begin{array}{c}460 \\
35.30 \% \\
41 \\
3.15 \% \\
74 \\
5.68 \%\end{array}$ & $\begin{array}{c}211 \\
16.19 \% \\
27 \\
2.07 \% \\
41 \\
3.15 \%\end{array}$ & $\begin{array}{c}94 \\
7.21 \% \\
11 \\
0.84 \% \\
34 \\
2.61 \%\end{array}$ & $\begin{array}{c}39 \\
2.99 \% \\
6 \\
0.46 \% \\
12 \\
0.92 \%\end{array}$ & $\begin{array}{c}43 \\
3.30 \% \\
9 \\
0.69 \% \\
15 \\
1.15 \%\end{array}$ & $\begin{array}{c}133 \\
10.21 \% \\
24 \\
1.84 \% \\
29 \\
2.23 \%\end{array}$ & 24.69 & 10 & 0.006 \\
\hline $\begin{array}{l}\text { Mathematic } \\
\mathrm{s} \text { classes } \\
\text { are boring }\end{array}$ & $\begin{array}{c}\text { High } \\
\text { Average } \\
\text { Low }\end{array}$ & $\begin{array}{c}175 \\
13.43 \% \\
18 \\
1.38 \% \\
30 \\
2.30 \%\end{array}$ & $\begin{array}{c}66 \\
5.07 \% \\
7 \\
0.54 \% \\
14 \\
1.07 \%\end{array}$ & $\begin{array}{c}80 \\
6.14 \% \\
13 \\
1.00 \% \\
25 \\
1.92 \%\end{array}$ & $\begin{array}{c}66 \\
5.07 \% \\
7 \\
0.54 \% \\
26 \\
2.00 \% \\
\end{array}$ & $\begin{array}{c}121 \\
9.29 \% \\
25 \\
1.92 \% \\
23 \\
1.77 \%\end{array}$ & $\begin{array}{c}472 \\
36.22 \% \\
48 \\
3.68 \% \\
87 \\
6.68 \% \\
\end{array}$ & 22.45 & 10 & 0.013 \\
\hline
\end{tabular}

Table 4 shows students' attitude towards learning mathematics. Results show that students with higher working memory capacity feel more confidence in mathematics classes $\left(\chi^{2}=22.169, \mathrm{p}=0.014,58.4 \%\right)$, consider mathematics easy $\left(\chi^{2}=23.76, \mathrm{p}=0.008,32.9 \%\right)$, think that they are getting better in mathematics $\left(\chi^{2}=30.68, \mathrm{p}=0.001,49.5 \%\right)$, coping well $\left(\chi^{2}=24.69, \mathrm{p}=0.006,51.5 \%\right)$ and feel that mathematics classes are interesting $\left(\chi^{2}=22.45, \mathrm{p}=0.013,45.5 \%\right)$ more than the students' with average and low working memory capacity.

Table 5

Chi-square for the comparison of students' mathematics preference among other subjects on the basis of their working memory capacity

\begin{tabular}{|c|c|c|c|c|c|c|c|c|c|c|}
\hline \multirow{3}{*}{$\begin{array}{l}\text { Subjects } \\
\text { Pak } \\
\text { Studies }\end{array}$} & \multirow{2}{*}{$\begin{array}{c}\text { Working } \\
\text { Memory }\end{array}$} & \multicolumn{5}{|c|}{$\begin{array}{c}\text { Frequencies } \\
\%\end{array}$} & \multirow{2}{*}{$\begin{array}{c}\begin{array}{c}\text { Subje } \\
\text { cts }\end{array} \\
\text { Maths }\end{array}$} & \multirow{2}{*}{$\frac{\chi^{2}}{25.66}$} & \multirow{2}{*}{$\frac{d f}{10}$} & \multirow{2}{*}{$\begin{array}{c}\begin{array}{c}p- \\
\text { value }\end{array} \\
0.004\end{array}$} \\
\hline & & 269 & 53 & 34 & 28 & 528 & & & & \\
\hline & & $20.64 \%$ & $4.07 \%$ & $2.61 \%$ & $2.15 \%$ & $5.22 \% 40.52 \%$ & & & & \\
\hline & Average & 34 & 6 & 3 & 5 & 53 & & & & \\
\hline & & $2.61 \%$ & $0.46 \%$ & $0.23 \%$ & $0.38 \%$ & $1.30 \% 4.07 \%$ & & & & \\
\hline & Low & $\begin{array}{c}63 \\
4.83 \%\end{array}$ & $\begin{array}{c}8 \\
0.61 \%\end{array}$ & $\begin{array}{c}17 \\
1.30 \%\end{array}$ & $\begin{array}{c}8 \\
0.61 \%\end{array}$ & $\begin{array}{cc}20 & 89 \\
1.53 \% & 6.83 \%\end{array}$ & & & & \\
\hline Maths & High & 279 & 38 & 29 & 37 & 535 & English & 42.30 & 10 & 0.000 \\
\hline & & $21.41 \%$ & $2.92 \%$ & $2.23 \%$ & $2.84 \%$ & $4.76 \% 41.06 \%$ & & & & \\
\hline & Average & $\begin{array}{c}29 \\
2.23 \%\end{array}$ & $\begin{array}{c}6 \\
0.46 \%\end{array}$ & $\begin{array}{c}5 \\
0.38 \%\end{array}$ & $\begin{array}{c}9 \\
0.69 \%\end{array}$ & $\begin{array}{cc}13 & 56 \\
1.00 \% & 4.30 \%\end{array}$ & & & & \\
\hline
\end{tabular}




\begin{tabular}{|c|c|c|c|c|c|c|c|c|c|c|}
\hline & Low & 48 & 12 & 22 & 16 & 14 & 93 & & & \\
\hline & & $3.68 \%$ & $0.92 \%$ & $1.69 \%$ & $1.23 \%$ & $1.07 \%$ & $7.14 \%$ & & & \\
\hline \multirow[t]{5}{*}{ Maths } & High & $\begin{array}{c}255 \\
19.57 \%\end{array}$ & $\begin{array}{c}32 \\
2.46 \%\end{array}$ & $\begin{array}{c}36 \\
2.76 \%\end{array}$ & $\begin{array}{c}37 \\
2.84 \%\end{array}$ & $\begin{array}{c}50 \\
3.84 \%\end{array}$ & $\begin{array}{c}570 \\
43.75 \%\end{array}$ & $\begin{array}{l}\text { Islamic } \\
\text { studies }\end{array}$ & 29.9310 & 0.001 \\
\hline & Average & 20 & 6 & 5 & 9 & 8 & 70 & & & \\
\hline & & $1.53 \%$ & $0.46 \%$ & $0.38 \%$ & $0.69 \%$ & $0.61 \%$ & $5.37 \%$ & & & \\
\hline & Low & 33 & 12 & 17 & 14 & 16 & 113 & & & \\
\hline & & $2.53 \%$ & $0.92 \%$ & $1.30 \%$ & $1.07 \%$ & $1.23 \%$ & $8.67 \%$ & & & \\
\hline \multirow[t]{6}{*}{ Physics } & High & 412 & 59 & 46 & 42 & 53 & 368 & Maths & 23.2510 & 0.010 \\
\hline & & $31.62 \%$ & $4.53 \%$ & $3.53 \%$ & $3.22 \%$ & $4.07 \% 2$ & $28.24 \%$ & & & \\
\hline & Average & 46 & 12 & 2 & 3 & 13 & 42 & & & \\
\hline & & $3.53 \%$ & $0.92 \%$ & $0.15 \%$ & $0.23 \%$ & $1.00 \%$ & $3.22 \%$ & & & \\
\hline & Low & 79 & 11 & 16 & 17 & 13 & 69 & & & \\
\hline & & $6.06 \%$ & $0.84 \%$ & $1.23 \%$ & $1.30 \%$ & $1.00 \%$ & $5.30 \%$ & & & \\
\hline
\end{tabular}

Table 5 shows that there is a significant difference between students' preferences about mathematics among other subjects. Students with higher working memory capacity prefer mathematics over Pakistan Studies $\left(\chi^{2}\right.$ $=25.66, \mathrm{p}=0.004,45.7 \%)$, while English $\left(\chi^{2}=42.30, \mathrm{p}=0.000,45.8 \%\right)$, Islamic studies $\left(\chi^{2}=29.93, \mathrm{p}=0.001,47.6 \%\right)$ and physics $\left(\chi^{2}=23.25\right.$, $\mathrm{p}=0.010,36.2 \%)$ were preferred over mathematics more than the students' with average and low working memory.

Table 6

Chi-square for the comparison of students' attitude towards different topics based on their working memory capacity

\begin{tabular}{|c|c|c|c|c|c|c|c|c|c|c|}
\hline \multirow{2}{*}{$\frac{\text { Subjects }}{\text { Fraction }}$} & \multirow{2}{*}{$\begin{array}{l}\text { Working } \\
\text { Memory } \\
\text { High }\end{array}$} & \multicolumn{5}{|c|}{$\begin{array}{c}\text { Frequencies } \\
\%\end{array}$} & Subjects & $\chi^{2}$ & $\mathrm{df}$ & $\begin{array}{c}p- \\
\text { value }\end{array}$ \\
\hline & & $\begin{array}{c}422 \\
32.39 \%\end{array}$ & $\begin{array}{c}60 \\
4.60 \%\end{array}$ & $\begin{array}{c}50 \\
3.84 \%\end{array}$ & $\begin{array}{c}21 \\
1.61 \%\end{array}$ & $\begin{array}{cc}49 & 378 \\
3.76 \% 29.01 \%\end{array}$ & Geometry & 28.66 & 10 & 0.001 \\
\hline & Average & 52 & 7 & 3 & 5 & $9 \quad 42$ & & & & \\
\hline & & $3.99 \%$ & $0.54 \%$ & $0.23 \%$ & $0.38 \%$ & $0.69 \% 3.22 \%$ & & & & \\
\hline & Low & $\begin{array}{c}64 \\
4.91 \%\end{array}$ & $\begin{array}{c}16 \\
1.23 \%\end{array}$ & $\begin{array}{c}25 \\
1.92 \%\end{array}$ & $\begin{array}{c}5 \\
0.38 \%\end{array}$ & $\begin{array}{cc}8 & 87 \\
0.61 \% 6.68 \%\end{array}$ & & & & \\
\hline Sets & High & $\begin{array}{c}615 \\
47.20 \%\end{array}$ & $\begin{array}{c}89 \\
6.83 \%\end{array}$ & $\begin{array}{c}44 \\
3.38 \%\end{array}$ & $\begin{array}{c}22 \\
1.69 \%\end{array}$ & $\begin{array}{cc}36 & 174 \\
2.76 \% 13.35 \%\end{array}$ & Fractions & 20.23 & 10 & 0.027 \\
\hline & Average & $\begin{array}{c}59 \\
4.53 \%\end{array}$ & $\begin{array}{c}21 \\
1.61 \%\end{array}$ & $\begin{array}{c}4 \\
0.31 \%\end{array}$ & $\begin{array}{c}5 \\
0.38 \%\end{array}$ & $\begin{array}{cc}3 & 26 \\
0.23 \% 2.00 \%\end{array}$ & & & & \\
\hline & Low & 114 & 20 & 14 & 7 & $12 \quad 38$ & & & & \\
\hline & & $8.75 \%$ & $1.53 \%$ & $1.07 \%$ & $0.54 \%$ & $0.92 \% 2.92 \%$ & & & & \\
\hline
\end{tabular}




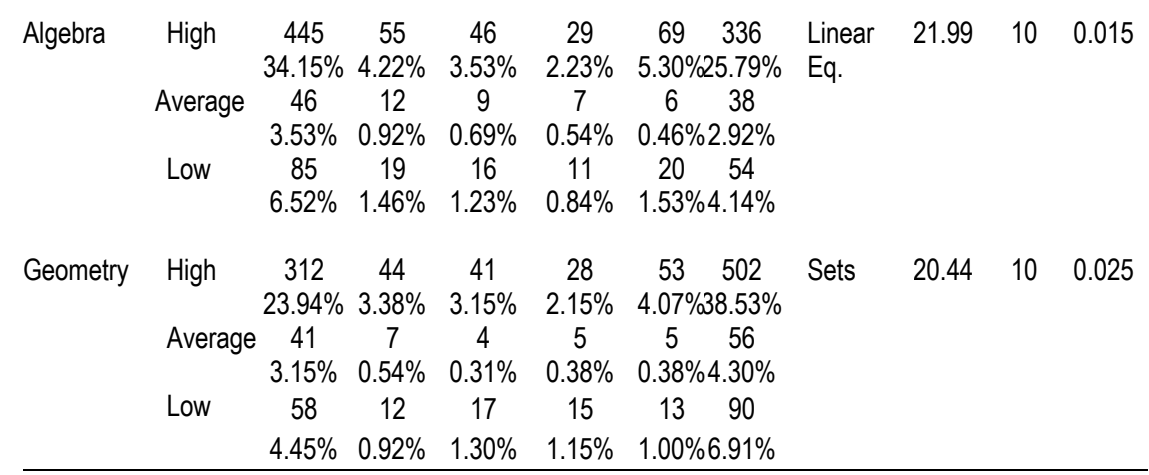

In table 6 students' attitude towards different topics of mathematics is presented. There is a significant difference between students' attitude towards different topics. Results in table 6 show that students with high working capacity prefer fractions over Geometry $\left(\chi^{2}=28.66, p=0.001\right.$, $37.0 \%)$, sets over fractions $\left(\chi^{2}=20.23, \mathrm{p}=0.027,54.0 \%\right)$, algebra over linear equations $\left(\chi^{2}=21.99, \mathrm{p}=0.015,38.4 \%\right)$, sets over geometry $\left(\chi^{2}\right.$ $=20.44, \mathrm{p}=0.025,42.6 \%$ ).

Table 7

Chi-square for the comparison of students' opinion about mathematics as a subject on the basis of their working memory capacity

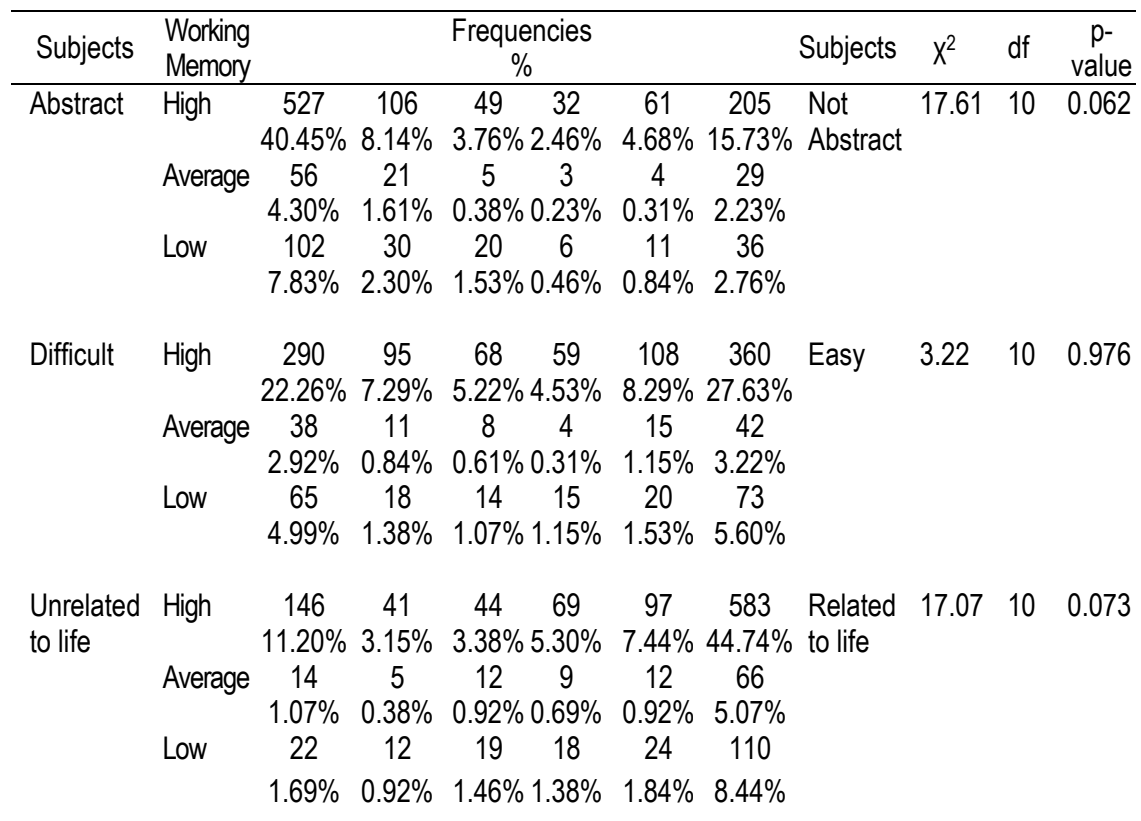




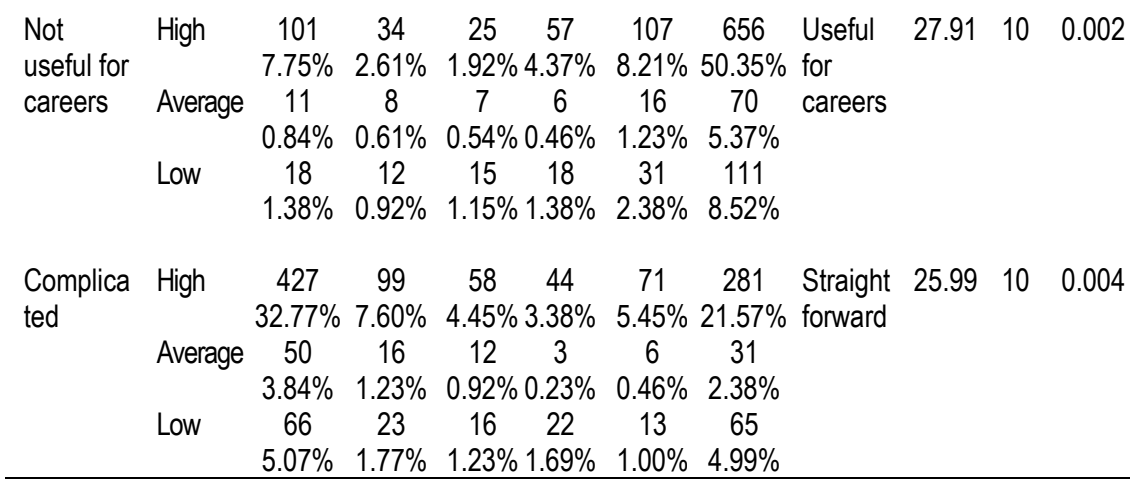

Table 7 represents that there is no significant difference among high, average and low working memory students' opinion about the abstractness $\left(\chi^{2}\right.$ $=17.609, \mathrm{p}=0.062)$, difficulty $\left(\chi^{2}=3.22, \mathrm{p}=0.976\right)$ and relatedness of mathematics to life $\left(\chi^{2}=17.07, \mathrm{p}=0.073\right)$. But there is a significant difference between students' opinion about the usefulness of mathematics in career $\left(\chi^{2}\right.$ $=27.91, \mathrm{p}=0.002,58.6 \%)$ and complicatedness $\left(\chi^{2}=25.99, \mathrm{p}=0.004,40.4 \%\right)$ of mathematics. Further results revealed that students with high working memory capacity think mathematics more useful in their careers than the students with average and low working memory capacity. Similarly, higher working memory students perceive mathematics more complicated than average and low working memory students.

Table 8

Chi-square for the comparison of science students' opinion about mathematics lessons on the basis of their working memory capacity

\begin{tabular}{llcccccccc}
\hline Statement & $\begin{array}{l}\text { Working } \\
\text { Memory }\end{array}$ & Always & Often & Sometimes & Rarely & Never & $\chi^{2}$ & $\begin{array}{c}\text { df } \\
\text { value }\end{array}$ \\
\hline I do not & High & 47 & 109 & 225 & 423 & 176 & 8.20 & 8 & 0.414 \\
understand & & $3.61 \%$ & $8.37 \%$ & $17.27 \%$ & $32.46 \%$ & $13.51 \%$ & & & \\
what is & Average & 6 & 13 & 29 & 48 & 22 & & & \\
taught & & $0.46 \%$ & $1.00 \%$ & $2.23 \%$ & $3.68 \%$ & $1.69 \%$ & & & \\
& Low & 18 & 26 & 53 & 75 & 33 & & & \\
& & $1.38 \%$ & $2.00 \%$ & $4.07 \%$ & $5.76 \%$ & $2.53 \%$ & & & \\
& & & & & & & & & \\
I find doing & High & 143 & 251 & 223 & 234 & 129 & 8.33 & 8 & 0.402 \\
mathematics & & $10.97 \%$ & $19.26 \%$ & $17.11 \%$ & $17.96 \%$ & $9.90 \%$ & & & \\
problems & Average & 21 & 39 & 24 & 20 & 14 & & & \\
repetitive & & $1.61 \%$ & $2.99 \%$ & $1.84 \%$ & $1.53 \%$ & $1.07 \%$ & & & \\
& Low & 34 & 46 & 47 & 55 & 23 & & & \\
& & $2.61 \%$ & $3.53 \%$ & $3.61 \%$ & $4.22 \%$ & $1.77 \%$ & & &
\end{tabular}




\begin{tabular}{|c|c|c|c|c|c|c|c|c|c|}
\hline $\begin{array}{l}\text { The } \\
\text { explanations }\end{array}$ & High & $\begin{array}{c}46 \\
3.53 \%\end{array}$ & $\begin{array}{c}176 \\
13.51 \%\end{array}$ & $\begin{array}{c}244 \\
18.73 \%\end{array}$ & $\begin{array}{c}303 \\
23.25 \%\end{array}$ & $\begin{array}{c}211 \\
16.19 \%\end{array}$ & 13.99 & 8 & 0.082 \\
\hline $\begin{array}{l}\text { are not } \\
\text { clear }\end{array}$ & $\begin{array}{l}\text { Average } \\
\text { Low }\end{array}$ & $\begin{array}{c}6 \\
0.46 \% \\
12 \\
0.92 \%\end{array}$ & $\begin{array}{c}17 \\
1.30 \% \\
42 \\
3.22 \%\end{array}$ & $\begin{array}{c}26 \\
2.00 \% \\
46 \\
3.53 \%\end{array}$ & $\begin{array}{c}51 \\
3.91 \% \\
75 \\
5.76 \%\end{array}$ & $\begin{array}{c}18 \\
1.38 \% \\
30 \\
2.30 \%\end{array}$ & & & \\
\hline $\begin{array}{l}\text { I am not } \\
\text { sure what } \\
\text { should be } \\
\text { doing }\end{array}$ & $\begin{array}{l}\text { High } \\
\text { Average } \\
\text { Low }\end{array}$ & $\begin{array}{c}49 \\
3.76 \% \\
10 \\
0.77 \% \\
12 \\
0.92 \%\end{array}$ & $\begin{array}{c}185 \\
14.20 \% \\
12 \\
0.92 \% \\
37 \\
2.84 \%\end{array}$ & $\begin{array}{c}204 \\
15.66 \% \\
30 \\
2.30 \% \\
51 \\
3.91 \%\end{array}$ & $\begin{array}{c}348 \\
26.71 \% \\
41 \\
3.15 \% \\
70 \\
5.37 \%\end{array}$ & $\begin{array}{c}194 \\
14.89 \% \\
25 \\
1.92 \% \\
35 \\
2.69 \%\end{array}$ & 9.82 & 8 & 0.278 \\
\hline $\begin{array}{l}\text { I find I } \\
\text { make } \\
\text { many } \\
\text { mistakes }\end{array}$ & $\begin{array}{l}\text { High } \\
\text { Average } \\
\text { Low }\end{array}$ & $\begin{array}{c}102 \\
7.83 \% \\
9 \\
0.69 \% \\
17 \\
1.30 \%\end{array}$ & $\begin{array}{c}210 \\
16.12 \% \\
25 \\
1.92 \% \\
51 \\
3.91 \%\end{array}$ & $\begin{array}{c}250 \\
19.19 \% \\
32 \\
2.46 \% \\
47 \\
3.61 \%\end{array}$ & $\begin{array}{c}323 \\
24.79 \% \\
43 \\
3.30 \% \\
67 \\
5.14 \%\end{array}$ & $\begin{array}{c}95 \\
7.29 \% \\
9 \\
0.69 \% \\
23 \\
1.77 \%\end{array}$ & 4.40 & 8 & 0.819 \\
\hline $\begin{array}{l}\text { There is } \\
\text { too much } \\
\text { homework }\end{array}$ & $\begin{array}{l}\text { High } \\
\text { Average } \\
\text { Low }\end{array}$ & $\begin{array}{c}248 \\
19.03 \% \\
32 \\
2.46 \% \\
44 \\
3.38 \%\end{array}$ & $\begin{array}{c}216 \\
16.58 \% \\
26 \\
2.00 \% \\
31 \\
2.38 \% \\
\end{array}$ & $\begin{array}{c}166 \\
12.74 \% \\
21 \\
1.61 \% \\
44 \\
3.38 \% \\
\end{array}$ & $\begin{array}{c}209 \\
16.04 \% \\
26 \\
2.00 \% \\
47 \\
3.61 \%\end{array}$ & $\begin{array}{c}141 \\
10.82 \% \\
13 \\
1.00 \% \\
39 \\
2.99 \% \\
\end{array}$ & 11.11 & 8 & 0.196 \\
\hline
\end{tabular}

Table 8 shows that chi square values are not significant for students' opinion about the understanding what is taught $\left(\chi^{2}=8.20, \mathrm{p}=0.414\right)$, find doing mathematics problems repetitive $\left(\chi^{2}=8.33, \mathrm{p}=0.402\right)$, explanations are not clear $\left(\chi^{2}=13.99, \mathrm{p}=0.082\right)$, not sure what should be doing $\left(\chi^{2}=9.82\right.$, $\mathrm{p}=0.278)$, find making many mistakes $\left(\chi^{2}=4.40, \mathrm{p}=0.819\right)$ and having too much homework $\left(\chi^{2}=11.11, \mathrm{p}=0.196\right)$. So we can conclude that there is no significant difference among high, average and low working memory students' opinions about the mathematics classes. Further low working memory capacity students' feel little opportunity to explain things in mathematics test or examination than students with high and average working memory capacity. 
Table 9

Chi-square for the comparison of science students' opinion about mathematics test and examination on the basis of their working memory capacity

\begin{tabular}{|c|c|c|c|c|c|c|c|c|c|}
\hline Statement & $\begin{array}{l}\text { Working } \\
\text { Memory }\end{array}$ & Always & Often & Sometimes & Rarely & Never & $x^{2}$ & $\mathrm{df}$ & $\begin{array}{l}\mathrm{p}- \\
\text { value }\end{array}$ \\
\hline $\begin{array}{l}\text { I tend to } \\
\text { panic with } \\
\text { difficult } \\
\text { problems }\end{array}$ & $\begin{array}{l}\text { High } \\
\text { Average } \\
\text { Low }\end{array}$ & $\begin{array}{c}144 \\
11.05 \% \\
18 \\
1.38 \% \\
36 \\
2.76 \%\end{array}$ & $\begin{array}{c}180 \\
13.81 \% \\
21 \\
1.61 \% \\
50 \\
3.84 \%\end{array}$ & $\begin{array}{c}198 \\
15.20 \% \\
20 \\
1.53 \% \\
37 \\
2.84 \%\end{array}$ & $\begin{array}{c}301 \\
23.10 \% \\
44 \\
3.38 \% \\
61 \\
4.68 \%\end{array}$ & $\begin{array}{c}157 \\
12.05 \% \\
15 \\
1.15 \% \\
21 \\
1.61 \%\end{array}$ & 10.93 & 8 & 0.206 \\
\hline $\begin{array}{l}\text { They } \\
\text { involve a lot } \\
\text { of revision } \\
\text { the day } \\
\text { before }\end{array}$ & $\begin{array}{l}\text { High } \\
\text { Average } \\
\text { Low }\end{array}$ & $\begin{array}{c}268 \\
20.57 \% \\
37 \\
2.84 \% \\
62 \\
4.76 \%\end{array}$ & $\begin{array}{c}220 \\
16.88 \% \\
27 \\
2.07 \% \\
38 \\
2.92 \%\end{array}$ & $\begin{array}{c}159 \\
12.20 \% \\
21 \\
1.61 \% \\
50 \\
3.84 \%\end{array}$ & $\begin{array}{c}190 \\
14.58 \% \\
24 \\
1.84 \% \\
32 \\
2.46 \%\end{array}$ & $\begin{array}{c}143 \\
10.97 \% \\
9 \\
0.69 \% \\
23 \\
1.77 \%\end{array}$ & 14.74 & 8 & 0.064 \\
\hline $\begin{array}{l}\text { I find I am } \\
\text { short of time }\end{array}$ & $\begin{array}{l}\text { High } \\
\text { Average } \\
\text { Low }\end{array}$ & $\begin{array}{c}278 \\
21.34 \% \\
35 \\
2.69 \% \\
60 \\
4.60 \%\end{array}$ & $\begin{array}{c}227 \\
17.42 \% \\
26 \\
2.00 \% \\
43 \\
3.30 \%\end{array}$ & $\begin{array}{c}185 \\
14.20 \% \\
24 \\
1.84 \% \\
45 \\
3.45 \%\end{array}$ & $\begin{array}{c}189 \\
14.50 \% \\
24 \\
1.84 \% \\
34 \\
2.61 \%\end{array}$ & $\begin{array}{c}101 \\
7.75 \% \\
9 \\
0.69 \% \\
23 \\
1.77 \%\end{array}$ & 3.12 & 8 & 0.927 \\
\hline $\begin{array}{l}\text { I often make } \\
\text { mistakes }\end{array}$ & $\begin{array}{l}\text { High } \\
\text { Average }\end{array}$ & $\begin{array}{c}98 \\
7.52 \% \\
11 \\
0.84 \% \\
30 \\
2.30 \%\end{array}$ & $\begin{array}{c}205 \\
15.73 \% \\
25 \\
1.92 \% \\
34 \\
2.61 \%\end{array}$ & $\begin{array}{c}250 \\
19.19 \% \\
26 \\
2.00 \% \\
47 \\
3.61 \%\end{array}$ & $\begin{array}{c}347 \\
26.63 \% \\
47 \\
3.61 \% \\
71 \\
5.45 \%\end{array}$ & $\begin{array}{c}80 \\
6.14 \% \\
9 \\
0.69 \% \\
23 \\
1.77 \%\end{array}$ & 8.75 & 8 & 0.364 \\
\hline $\begin{array}{l}\text { I cannot } \\
\text { remember } \\
\text { how to do } \\
\text { things }\end{array}$ & $\begin{array}{l}\text { High } \\
\text { Average } \\
\text { Low }\end{array}$ & $\begin{array}{c}90 \\
6.91 \% \\
13 \\
1.00 \% \\
19 \\
1.46 \%\end{array}$ & $\begin{array}{c}165 \\
12.66 \% \\
18 \\
1.38 \% \\
35 \\
2.69 \%\end{array}$ & $\begin{array}{c}184 \\
14.12 \% \\
22 \\
1.69 \% \\
45 \\
3.45 \%\end{array}$ & $\begin{array}{c}334 \\
25.63 \% \\
41 \\
3.15 \% \\
74 \\
5.68 \%\end{array}$ & $\begin{array}{c}207 \\
15.89 \% \\
24 \\
1.84 \% \\
32 \\
2.46 \%\end{array}$ & 4.23 & 8 & 0.836 \\
\hline $\begin{array}{l}\text { There is } \\
\text { little } \\
\text { opportunity } \\
\text { to explain } \\
\text { things }\end{array}$ & $\begin{array}{l}\text { High } \\
\text { Average } \\
\text { Low }\end{array}$ & $\begin{array}{c}87 \\
6.68 \% \\
12 \\
0.92 \% \\
19 \\
1.46 \%\end{array}$ & $\begin{array}{c}192 \\
14.74 \% \\
21 \\
1.61 \% \\
39 \\
2.99 \%\end{array}$ & $\begin{array}{c}218 \\
16.73 \% \\
23 \\
1.77 \% \\
49 \\
3.76 \%\end{array}$ & $\begin{array}{c}278 \\
21.34 \% \\
42 \\
3.22 \% \\
76 \\
5.83 \%\end{array}$ & $\begin{array}{c}205 \\
15.73 \% \\
20 \\
1.53 \% \\
22 \\
1.69 \%\end{array}$ & 15.99 & 8 & 0.043 \\
\hline
\end{tabular}

Similarly, table 9 shows that there is no significant difference among students' opinion about tends to panic with difficult problems $\left(\chi^{2}=10.93\right.$, 
$\mathrm{p}=0.206)$, involve a lot of revision the day before $\left(\chi^{2}=14.74, \mathrm{p}=0.064\right)$, find short of time $\left(\chi^{2}=3.12, p=0.927\right)$, often make mistakes $\left(\chi^{2}=8.75\right.$, $\mathrm{p}=0.364)$, cannot remember how to do things $\left(\chi^{2}=4.23, \mathrm{p}=0.836\right)$. But there is a significant difference between their feelings about little opportunity to explain things in mathematics test and examination $\left(\chi^{2}\right.$ $=15.99, \mathrm{p}=0.043,37.2 \%$ ).

\section{Conclusions and Discussion}

The purpose of the research was to discover the working memory capacity of the students and find its effect on their attitude towards mathematics at secondary level. On the basis of the results we can conclude that on the average students at secondary level had good working memory capacity i.e. 6.68. Alenzi (2008) argued this level as in accordance to the set standards for this age group which is round about 14 years and gives a mean of little over 6 . Most of the students possess high working memory capacity. There was a small difference among students' working memory capacity. However, female students had better working memory capacity than male students, urban students had better working memory capacity than rural students and the biology students had high working memory capacity than computer science students. But the students of $9^{\text {th }} \& 10^{\text {th }}$ grades, and public \& private schools had no difference between their working memory capacities.

When working memory capacity is high, it proposes a benefit in attaining success in mathematics and the success is correlated to positive attitude (Alenezi, 2008). The results of the present study are consistent with Alenezi (2008) findings as the analysis of high average and low working memory capacity students' attitude towards mathematics revealed that students with high and average working memory capacity had better attitude and feeling in general about mathematics than the students with low working memory capacity. For example, the students with average and high working memory capacity enjoy mathematics more than the students with low working memory capacity. Also, high working memory students had better views about mathematics as a useful subject and its usefulness in daily life than the average and low working memory capacity students. Although, there was no difference among students' views about understanding the mathematics ideas easily, everyone should learn mathematics at secondary level, students thinking that they are good in mathematics and views about spending more time for studying mathematics. But again, percentage of responses showed that high 
working memory students had better views than average and low working memory students.

It was also concluded that students with high working memory capacity had better attitude towards learning mathematics than average and low working memory students. High working memory students had confidence in mathematics classes, think mathematics is easy, felt they were getting better in mathematics, coping well and considered mathematics classes interesting more than average and low working memory capacity students.

Similarly, the high working memory capacity students prefer mathematics over Pakistan Studies, but they prefer English, Islamic studies and Physics over mathematics. The students with high working memory capacity prefer sets over fractions and geometry more than the average and low working memory capacity students. So, it can be concluded that high working capacity students' better attitude towards abstract topics of mathematics. There was no difference among high, average and low working memory capacity students' attitude towards mathematics as an abstract, easy and related to life subject. The high working memory students' perceived mathematics more useful in their careers and more complicated subject than average and low working memory capacity students.

The opinion of the students about lesson and test or examination of mathematics was same. However, percentage of responses revealed that high working memory students had better attitude towards mathematics lessons, tests and exams. Finally, we can conclude that high working memory capacity causes satisfaction and better feelings about mathematics and develops positive attitude for learning mathematics as Jung and Reid (2009) suggested that working memory capacity is likely to be more correlated with affective elements of attitude than more cognitive aspects. 


\section{References}

Adams, J. W., \& Hitch, G. J. (1997). Working memory and children's mental addition. Journal of Experimental Child Psychology, 67, 2138 .

Alenezi, D. F. (2004). Difficulties associated with teaching and learning mathematics: A study of psychological factors affecting pupils performance (Unpublished Master Thesis). Science Education Centre, Glasgow, University of Glasgow.

Alenezi, D. F. (2008). A study of learning mathematics related to some cognitive factors and to attitudes (Unpublished doctoral dissertation). Faculty of Education, University of Glasgow.

Ali, A. A., \& Reid, N. (2012). Understanding mathematics: Some key factors. European Journal of Educational Research, 1(3), 283-299.

Arslan, C., Yavuza, G., \& Deringol-Karatasa, Y. (2014). Attitudes of elementary school students towards solving mathematics problems. Procedia - Social and Behavioral Sciences, 152, 557-562.

Atkinson, R. C., \& Shiffrin, R. M. (1968). Human memory: A proposed system and its component processes. In K. W. Spence \& J. T. Spence (Eds.), The psychology of learning and motivation: Advances in research and theory ( $2^{\text {nd }}$ ed., pp. 89- 195). New York: Academic Press.

Baddeley, A. D., \& Hitch, G. (1974). Working memory. In G. H. Bower (Ed.), The Psychology of Learning and Motivation: Advances in Research and Theory (pp. 47-89). New York: Academic Press.

Bull, R., \& Espy, K. A. (2006). Working memory, executive functioning, and children's mathematics. In S. Pickering (Ed.), Working memory and education (pp. 93-123). New York: Academic Press.

Bull, R., \& Scerif, G. (2001). Executive functioning as a predictor of children's mathematics ability: Inhibition, task switching, and working memory. Development Neuropsychology, 19, 273-293.

Christou, K. (2001). Difficulties in solving algebra story problems with secondary pupils. Glasgow: Science Education Centre, University of Glasgow. 
Holmes, J., \& Adams, J. W. (2006). Working memory and children's mathematics skills: Implications for mathematical development and mathematics curricula. Educational Psychology, 26(3), 339-366.

James, W. (1970). The principles of psychology ( $1^{\text {st }}$ vol.). New York: Holt.

Jung, S. E., \& Reid, N. (2009). Working memory and attitudes. Research in Science \& Technological Education, 27(2), 205-223.

Jarvis, H. L., \& Gathercole, S. E. (2003). Verbal and nonverbal working memory and achievements on national curriculum tests at 7 and 14 years of age. Educational and Child Psychology, 20, 123-140.

Ma, X., \& Kishor, N. (1997). Assessing the relationship between attitude toward mathematics and achievement in mathematics: A metaanalysis. Journal for Research in Mathematics Education, 28(1), 2647.

Marchis, I. (2013). Relation between students' attitude towards mathematics and their problem solving skills. PedActa, 3(2), 59-66.

Matthews, A., \& Pepper, D. (2005). Evaluation of participation in A-level mathematics: Interim report. London: Qualifications and Curriculum Agency.

Mclean, J., \& Hitch, G. J. (1999). Working memory impairments in children with specific arithmetic learning difficulties. Journal of Experimental Child Psychology, 74, 240-260.

McLeod, B. D. (1994). Research on affect and mathematics learning in the jrme: 1970 to the present. Journal for Research in Mathematics Education, 25(6), 637-647.

Mohd, N., Mahmood, T. F. P. T., \& Ismail, M. N. (2011). Factors that influence students in mathematics achievement. International Journal of Academic Research, 3(3), 49-54.

Pakistan Alliance for Maths and Science (2017). Powering Pakistan for the 21st Century; Vol II of III: The State of Maths and Science in Schools. Islamabad: Pakistan Alliance for Maths and Science.

Rubinstein, M. F. (1986). Tools for thinking and problem solving. New Jersey: Prentice Hall.

Simms, N. K., Frausel, R. R., \& Richland, L. E. (2018). Working memory predicts children's analogical reasoning. Journal of Experimental Child Psychology, 166, 160-177. 
Sternberg, R. J., \& Sternberg, K. (2012). Cognitive Psychology (6 $6^{\text {th }}$ Ed.). Belmont, CA: Wadsworth, Cengage Learning.

Swanson, H. L. (2016). Word problem solving, working memory and serious math difficulties: Do cognitive strategies really make a difference? Journal of Applied Research in Memory and Cognition, 5(4), 368-383.

Towse, J. N., \& Houston-Price, C. M. (2001). Combining representations in working memory: A brief report. British Journal of Developmental Psychology, 19, 319-324.

Citation of this Article:

Nazir, A., Rana, R. A., \& Khan, K. A. (2018). Effect of working memory on students' attitude towards mathematics at secondary level. Pakistan Journal of Education, 35(3), 97-118. 